\title{
Practical aspects of telehealth: financial considerations
}

\author{
P. K. Loh, ${ }^{1}$ S. Sabesan, ${ }^{2,3}$ D. Allen, ${ }^{5}$ P. Caldwell, ${ }^{8,9}$ R. Mozer, ${ }^{10}$ P. A. Komesaroff,,${ }^{11,12}$ P. Talman, ${ }^{13,14,15}$ \\ M. Williams ${ }^{4}$ N. Shaheen, ${ }^{6}$ O. Grabinski ${ }^{7}$ and D. Withnall' on behalf of The Royal Australasian College of \\ Physicians Telehealth Working Group
}

'Department of Geriatric Medicine, Royal Perth Hospital, Perth, Western Australia, ${ }^{2}$ School of Medicine and Dentistry, James Cook University, ${ }^{3}$ Department of Medical Oncology, Townsville Cancer Centre, Townsville, ${ }^{4}$ Department of Child and Adolescent Health, Mackay Hospital, Mackay, Queensland, ${ }^{5}$ Quality Occupational Health, ${ }^{6}$ Aged Care Services, Royal North Shore Hospital, ${ }^{7}$ Policy and Advocacy Unit, The Royal Australasian College of Physicians, Sydney, ${ }^{8}$ Discipline of Paediatrics and Child Health, ${ }^{9}$ Centre for Kidney Research, The Children's Hospital at Westmead Hospital, Sydney, ${ }^{10}$ Rehabilitation Medicine, Rankin Park Centre, Newcastle, New South Wales, ${ }^{11}$ Faculty of Medicine, ${ }^{12}$ Monash Centre for the Study of Ethics in Medicine and Society, ${ }^{13}$ Department of Epidemiology and Preventative Medicine, Monash University, Melbourne, ${ }^{14}$ Neurosciences Department, Geelong Hospital, ${ }^{15}$ Deakin Medical School, Geelong, Victoria, Australia

\author{
Key words \\ telehealth, Medicare telehealth incentive, video \\ conference consultation.

\section{Correspondence \\ Odette Grabinski, Policy and Advocacy Unit, The Royal Australasian College of Physicians, Sydney, NSW 2038, Australia. \\ Email: telehealth@racp.edu.au}

Received 24 March 2013; accepted 16 May 2013.

doi:10.1111/imj.12193

\begin{abstract}
The second in a series of articles about the practical aspects of telehealth, this paper includes information and a case history on the cost-benefits for patients and practitioners using telehealth. The case history demonstrates that telehealth can save travel time for patients, carers and specialists, and can reduce out-of-pocket expenses. The practical aspects of telehealth article series considers the contextual, clinical, technical and ethical components of online video consultations.
\end{abstract}

\section{Background}

Telehealth in the context of the Australian healthcare setting is the delivery of medical consultations using videoconferencing technology ${ }^{1,2}$ Video consultations can involve specialists and patients with general practitioners (GP) or allied health workers at the patient end, or with no medical support at the patient end. Telehealth has often been touted as a cost-effective way of providing services to patients who live a significant distance away from health services or have difficulty getting access to services because of physical disabilities. Earlier reviews have not shown cost-effectiveness of this modality. ${ }^{3,4} \mathrm{~A}$ later review by Wade et al. showed economic benefit for access to on-call specialist services and home care services but mixed benefit results for rural care. There was no economic benefit for the provision of primary care services between hospitals and GP. ${ }^{5}$ Access Economics estimated that there may be a benefit of $\$ 2-4$ billion if

Funding: None.

Conflict of interest: None. telehealth was widely adopted in Australia. ${ }^{6}$ There is often a high capital cost that should reduce with time as the technology becomes more accessible and affordable. Furthermore, the reduction in travel time for rural and remote patients may justify additional costs for some patients.

Two Australian studies have shown savings from the telehealth model of care in paediatrics and medical oncology, respectively, when the patient number was large. In the medical oncology telehealth model between Townsville and Mt. Isa where acute care was provided through telehealth, the resulting reduction in interhospital transfers enhanced the savings to the health system in addition to routine telehealth care. ${ }^{7,8}$ Two recent articles in the October 2012 issue of the Internal Medicine Journal add utility to this. Nagao et al. increased thrombolysis for stroke in Victoria using 'telestroke'. ${ }^{9}$ Roberts et al. succinctly discussed general telehealth issues in Australia then focused on the benefits of 'telerheumatology'. ${ }^{10}$

The Australian Government has provided Medicare items for telehealth service provision. The Royal Australasian College of Physicians telehealth Medicare 
Benefits Schedule (MBS) items and incentives fact sheet (Appendix 1) highlights the associated specialist telehealth consultation item numbers. For government hospital or a publicly funded organisation or agency employees, the funding may be based on activity-based funding, and the reimbursement may be different from the Medicare item numbers.

\section{Case history}

Mr JS is a 65-year-old man who wishes to be assessed for his driver's licence but lives in a country town $2.5 \mathrm{~h}$ by car to the nearest specialist neurologist, psychiatrist or geriatric medicine specialist. His GP is concerned about his short-term memory loss and needs some reassurance that Mr JS can have his driver's licence renewed. His GP organises a telehealth consultation with the relevant specialist, and Mr JS is present. After some discussion and mental state examination with Mr JS, it is agreed that $\mathrm{Mr}$ JS should have a driving assessment by an occupational therapist (OT) despite mild short-term memory loss. $\mathrm{Mr}$ JS has the OT assessment and is successful in renewing his driver's licence as his general driving skills and coordination are not significantly affected by the mild short-term memory loss.

\section{Commentary}

In this scenario, Mr JS is driven to his GP about $5 \mathrm{~min}$ away by his daughter for the 40-min telehealth consultation. The total time spent, including wait time, is about $1 \mathrm{~h}$. The physician is able to bill Medicare item 110 for consultation item with telehealth item 112 . The specialist physician does not have to travel out of his rooms, and his practice support staff arranges the consultation with the GP's practice support staff. Likewise, the GP is able to bill a Medicare consultation item as well as telehealth item. The daughter is able to continue her family and work commitments without too much disruption.

The setting whereby the specialist physician travels to the country town would require a 2.5 -h drive (a 5-h total for a return trip) and arrangements made for consultation rooms in the country town. The specialist in this scenario bills Medicare item 110 . The patient and his daughter only need to spend $\mathrm{l} \mathrm{h}$ of their time for the consultation including logistics and wait time. The GP would not be present, and the appointment may be delayed as the specialist may only travel to the country town on a weekly or monthly basis. Indeed, in many small rural towns where the patient number is small, outreach visits by specialist physicians may not occur at all.

If this patient travels to the specialist in the city, there would be a 5 -h return journey for the patient and his daughter. The time required may be in excess of $8 \mathrm{~h}$ with at least $\mathrm{l} \mathrm{h}$ in the specialist rooms, finding parking in the city, meals and refuelling stops for the country patient and his daughter. If she works, she would have to take time off; if she has children, she would need to arrange childcare or school pickups. The patient may be able to claim travel assistance from the State or Commonwealth Governments, but this is an additional cost. The GP is not present, and the attendance at the specialist surgery is dependent on the availability of his daughter either to get time off work or arrange childcare.

\section{Discussion}

In this case, the specialist can bill for Medicare items as per Appendix 1. The GP is also eligible to bill for the consultation, and in some cases, if an allied health practitioner is at the patient end of the consultation, they can also claim the Medicare video consultation items. ${ }^{11}$ The GP will monitor Mr JS's cognition and make a further referral to the specialist in 12 months if required. This consultation may be through telehealth initially to determine if further tests or face-to-face assessments are required.

Table 1 is a simple log of the travel time, waiting time and out-of-pocket costs pertinent to the specialist and patient based on their case history. The assumptions are that the utility face-to-face assessment is the same as a telehealth assessment. The cost of capital and resources to the healthcare system is not considered. This table is not a cost-benefit or a cost-effectiveness analysis where clinical outcomes are compared. Opportunity costs for the carer, patient and physician are not in the table, but it can be anticipated that the physician could be seeing patients during the 5-h travel time if he or she is not driving to see them face to face. There is potentially a loss of income for the carer who has taken time off to drive her father to and from the consultation.

\section{Conclusion}

Telehealth carries with it significant benefits for patients and their GPs and specialists, and in the future is likely to be appropriate for most patients at some point. Financial arrangements and logistic issues will help determine a patient's suitability for an online video consultation. This article outlines some of the factors for both patients and specialists that need to be taken into account by practitioners when they are assessing the balance between costs and benefits of telehealth in their own particular cases.

It is hoped that with greater understanding of the financial circumstances for telehealth in Australia, more physicians and patients will be amenable to this modality of service delivery. 
Table 1 Case history log of travel time \& expenses of clinical assessments face to face and telehealth

\begin{tabular}{|c|c|c|c|}
\hline & Rural assessment & Metropolitan assessment & Telehealth assessment \\
\hline Reimbursement & $\$$ & $\$$ & $\$$ \\
\hline Physician Medicare Item $110 \dagger$ & 128 & 128 & 128 \\
\hline Physician Telehealth Medicare Item $112 \dagger$ & 0 & 0 & 65 \\
\hline Subtotal & 128 & 128 & 193 \\
\hline \multicolumn{4}{|l|}{ Equipment } \\
\hline Transmission cost via internet per month $\ddagger$ & 0 & 0 & 70 \\
\hline $\begin{array}{l}\text { Personal computer }(\mathrm{PC}) \text { videoconference software package included in } \\
\text { practice IT bundle or accessed via Internet at no cost. }\end{array}$ & 0 & 0 & 0 \\
\hline Subtotal & 0 & 0 & 70 \\
\hline \multicolumn{4}{|l|}{ Travel cost } \\
\hline Physician§』 & 296 & 0 & 0 \\
\hline Patient and Carer Travel cost†† & 4 & 296 & 4 \\
\hline Subtotal & 300 & 296 & 4 \\
\hline \multicolumn{4}{|l|}{ Travel time } \\
\hline Physician travel time (return) in hoursłł & 5 & 0 & 0 \\
\hline Patient and carer travel time (return) in hours§§ & 0.1 & 5 & 0.1 \\
\hline $\begin{array}{l}\text { Patient and carer clinic time in hours (parking, patient transfer, waiting and } \\
\text { assessment Time) }\end{array}$ & 0.9 & 3 & 0.9 \\
\hline Total travel time in hours & 6 & 8 & 1 \\
\hline Expenses per patient AUD\$ञा & 428 & 424 & 267 \\
\hline
\end{tabular}

†Physician consultation Medicare Item 110 benefit is $\$ 151$ and the $85 \%$ benefit is $\$ 128$. Medicare Item 112 is $50 \%$ of the Item 110 reimbursable benefit at $85 \%$. Dollar figures are not exact and rounded off. ‡Telstra Bigpond Internet cable cost 50 gigabyte download per month (based on typical Internet service cost from Telstra Bigpond). §Physician sees the Telehealth patients in clinic in metropolitan area. There are no extra travel cost involved. In the country town, the physician loans the GP's consulting rooms at no cost, but in most situations, the physician may have to lease consultation rooms. $\{$ Estimated cost for $200-\mathrm{km}$ one-way car journey or $400-\mathrm{km}$ return at $\$ 0.74$ per kilometre based on Australian Taxation claim for 1.6- to 2.6-L car. Available at http://www.ato.gov.au/individuals/content.aspx?doc=/content/33874.htm ††Estimated cost for 2.5-km one-way car journey or $5 \mathrm{~km}$ return at $\$ 0.74$ per kilometre based on Australian Taxation claim for 1.6- to 2.6-L car. Available at ttp://www.ato.gov.au/individuals/content.aspx?doc=/content/33874.htm $\ddagger \ddagger$ One new clinical assessment per patient seen per hour per physician either by Telehealth or face to face implies that the physician could see five patients during the travel time. §§The patient and carer would qualify for patient travel assistance subsidies. ๆqThis is not a cost-effective or cost-benefit analysis. It is a summation of relevant expenses.

\section{Supplementary note}

The telehealth reimbursement itself may or may not be a financially viable alternative for a specialist practice depending on the circumstances of the physician. It would be useful to speak to the practice manager, accountant and practice partners before embarking on increasing telehealth activity. In most circumstances, it would be complementary to face-to-face services, and the Medicare reimbursement has a built-in compensation for additional administrative work in scheduling telehealth appointments. There is also a one off-capital payment for physician practitioners and residential aged care facilities that start telehealth services (Table 2). This allows the physician to obtain equipment, upgrade software or create a suitable telehealth environment in the practice premises.

Advice on Medicare telehealth initiatives and on accessing these payments is available on the Medicare website: http://www.mbsonline.gov.au/telehealth Information on the specific Medicare billing items is available at http://www.mbsonline.gov.au/telehealth Generally, the qualitative improvement in access to health care services for the patient and access to the patient by the specialist physician or GP may justify any additional resourcing to initiate and continue telehealth consultations.

Table 2 Australian Government telehealth financial incentives

\begin{tabular}{lcc}
\hline On-Board incentives & $2012-2013$ & $2013-2014$ \\
\hline Telehealth on-board (one off-payment paid in two instalments) & $\$ 4800$ & $\$ 3900$ \\
RACF on-board incentive (one off-payment paid in two instalments) & $\$ 4800$ & $\$ 3900$ \\
\hline
\end{tabular}

Source: http://www.mbsonline.gov.au/telehealth RACF, residential aged care facility. 


\section{References}

1 Physicians Telehealth Support Project. [homepage on the Internet]. Sydney: The Royal Australasian College of Physicians. [cited 2013 Jan]. Available from URL: http://www.racptelehealth. com.au

2 Sabesan S, Allen D, Loh PK, Caldwell P, Mozer R, Komesaroff PA et al. Practical aspects of telehealth: are my patients suited to telehealth? Intern Med J 2013; 43: 581-4.

3 Whitten PS, Mair FS, Haycox A, May CR, Williams TL, Hellmich S. Systematic review of cost effectiveness studies of telemedicine interventions. BMJ 2002; 324: 1434-7.

4 De La Torre A, Hernandez-Rodriguez C, Garcia L. Cost analysis in telemedicine: empirical evidence from sites in Arizona. $J$ Rural Health 2004; 20: 253-7.
5 Wade VA, Karnon J, Elshaugand AG, Hiller JE. A systematic review of economic analyses of telehealth services using real time video communication. BMC Health Serv Res 2010; 10: 233.

6 Access Economics for the Department of Broadband, Communications and the Digital Economy. 2010. Financial and externality impacts of high-speed broadband for telehealth. [cited 2013 Jan]; Available from URL: http:// www.archive.dbcde.gov.au/data/ assets/pdf_file/0019/130159/ Financialandexternalityimpactsofhighspeedbroadbandfortelehealth311.pdf

7 Smith AC, Scuffham P, Wootton $\mathrm{R}$. The costs and potential savings of a novel telepaediatric service in Queensland. BMC Health Serv Res 2007; 7: 35 .
8 Sabesan S, Brennan S. Teleoncology for cancer care in rural Australia. In: Graschew G, ed. Telemedicine Techniques and Applications. Tech Publishers; 2011; 289-306. Available from URL: http://www.intechopen.com

9 Nagao KJ, Koschel A, Haines HM, Bolitho LE, Yan B. Rural Victorian Telestroke project. Intern Med J 2012; 42: 1088-95.

10 Roberts LJ, Lamont EG, Lim I, Sabesan S, Barrett C. Telerheumatology: an idea whose time has come. Intern Med J 2012; 42: 1072-8.

11 MBS Video Consultation Items. [MBS Online page on the internet]. Canberra: Department of Health and Ageing. [cited 2013 May]. Available from URL: http://www.mbsonline.gov.au/ internet/mbsonline/publishing.nsf/ Content/connectinghealthservicesitemlist

\section{Appendix 1}

\section{Fact Sheet}

\section{MBS Items for Specialist Telehealth Consultations}

Telehealth MBS $^{11}$ items are available for telehealth consultations provided by specialists and consultant physicians.

To be eligible for these telehealth items, the patient must be in a regional or remote area (Australian Standard Geographical Classification (ASGC) remoteness areas (RA) RA2 or above) and be $15 \mathrm{kms}$ away from the specialist. To check ASGC classifications for locations around Australia and to determine if patients are eligible, physicians can visit www.doctorconnect.gov.au. Residents of residential aged care facilities and patients at Aboriginal Medical Services are also eligible, including those in metropolitan areas.

Telehealth consultations can be conducted with a general practitioner, nurse practitioner, practice nurse or Aboriginal Health Worker or other specialist supporting the patient. These are called 'supported consultations' and MBS items are available for the medical practitioner at the patient end. Specialists can conduct 'unsupported consultations' with patients, by connecting with patients directly via videoconference, and still claim the specialist end MBS items.

The Telehealth Specialist MBS items are:

Item Specialist Attendances

99 Professional attendance on a patient by a specialist practising in his or her specialty if:

(a) the attendance is by video conference; and

(b) the attendance is for a service:

(i) provided with item 104 lasting more than 10 minutes; or

(ii) provided with item 105; and

(c) the patient is not an admitted patient; and

(d) the patient:

(i) is located both:

(A) within a telehealth eligible area; and

(B) at the time of the attendance-at least $15 \mathrm{kms}$ by road from the specialist; or

(ii) is a care recipient in a residential care service; or

(iii) is a patient of:

(A) an Aboriginal Medical Service; or

(B) an Aboriginal Community Controlled Health Service;

for which a direction made under subsection 19 (2) of the Act applies.

Derived Fee: $50 \%$ of the fee for item 104 or 105 . Benefit: $85 \%$ of derived fee. 


\section{Consultant Physician}

Professional attendance on a patient by a consultant physician practising in his or her specialty if:

(a) the attendance is by video conference; and

(b) the attendance is for a service:

(i) provided with item 110 lasting more than 10 minutes; or

(ii) provided with item 116, 119, 132 or 133; and

(c) the patient is not an admitted patient; and

(d) the patient:

(i) is located both:

(A) within a telehealth eligible area; and

(B) at the time of the attendance-at least $15 \mathrm{kms}$ by road from the physician; or

(ii) is a care recipient in a residential care service; or

(iii) is a patient of:

(A) an Aboriginal Medical Service; or

(B) an Aboriginal Community Controlled Health Service;

for which a direction made under subsection 19 (2) of the Act applies.

Derived fee: $50 \%$ of the fee for the associated item. Benefit: $85 \%$ of derived fee.

\section{Geriatric Medicine - Consultant Physician or Specialist}

Professional attendance on a patient by a consultant physician or specialist practising in his or her specialty of geriatric medicine if:

(a) the attendance is by video conference; and

(b) item 141 or 143 applies to the attendance; and

(c) the patient is not an admitted patient; and

(d) the patient:

(i) is located both:

(A) within a telehealth eligible area; and

(B) at the time of the attendance-at least $15 \mathrm{kms}$ by road from the physician or specialist; or

(ii) is a care recipient in a residential care service; or

(iii) is a patient of:

(A) an Aboriginal Medical Service; or

(B) an Aboriginal Community Controlled Health Service;

for which a direction made under subsection 19 (2) of the Act applies.

Derived Fee: $50 \%$ of the fee for 141 or 143 . Benefit: $85 \%$ of derived fee.

\section{Consultation Occupational Physician Attendances}

Professional attendance by a consultant occupational physician practising in his or her specialty of occupational medicine:

(a) by video conference; and

(b) the attendance is for a service:

(i) provided with item 385 lasting more than 10 minutes; or

(ii) provided with item 386; and

(c) the patient is not an admitted patient; and

(d) the patient:

(i) is located both:

(A) within a telehealth eligible area; and

(B) at the time of the attendance-at least $15 \mathrm{kms}$ by road from the physician; or

(ii) is a care recipient in a residential care service; or

(iii) is a patient of:

(A) an Aboriginal Medical Service; or

(B) an Aboriginal Community Controlled Health Service;

for which a direction made under subsection 19 (2) of the Act applies

Derived Fee: $50 \%$ of the fee for the associated item. Benefit: $85 \%$ of derived fee. 
Loh et al.

Item Palliative Medicine Attendances

3015 Professional attendance on a patient by a specialist or consultant physician practising in his or her specialty of palliative medicine if:

(a) the attendance is by video conference; and

(b) the attendance is for a service:

(i) provided with item 3005 lasting more than 10 minutes; or

(ii) provided with item 3010 or 3014 ; and

(c) the patient is not an admitted patient; and

(d) the patient:

(i) is located both:

(A) within a telehealth eligible area; and

(B) at the time of the attendance-at least $15 \mathrm{kms}$ by road from the specialist or physician; or

(ii) is a care recipient in a residential care service; or

(iii) is a patient of:

(A) an Aboriginal Medical Service; or

(B) an Aboriginal Community Controlled Health Service;

for which a direction made under subsection 19 (2) of the Act applies

Derived Fee: $50 \%$ of the fee for item 3005,3010 or 3014 . Benefit: $85 \%$ of derived fee.

Source: www.mbsonline.gov.au

Physicians should note that if they are consulting with another specialist or subspecialist, the physician located with the patient should claim the MBS items for patient end consultations, which are usually claimed by general practitioners. The remote specialist or subspecialist can claim the specialist end items as normal. The patient end telehealth consultation numbers for telehealth consultations are:

- 2100, 2126, 2143, 2195 for consultations conducted from consulting rooms within a telehealth eligible area

- 2111, 2137, 2147, 2199 for consultations conducted from locations other than consultation rooms in a telehealth eligible area

- 2125, 2138, 2179, 2220 for consultations conducted from a residential aged care facility.

MBS items 113,114, 384, 3003 can also be claimed for initial specialist and consultant physician consultations of less than 10 minutes duration. Unlike the items above, these short consultation items are stand-alone items and do not need to be lodged with a derived fee item number.

\section{Telehealth Incentive Payments}

From now until mid-2014, an 'On-Board Incentive' payment is available for physicians who commence using telehealth. The incentive is paid in two instalments, the first after the first telehealth MBS item claim and the second after the tenth item claim is processed.

\begin{tabular}{lcc}
\hline Incentive & $2012-13$ & $2013-14$ \\
First telehealth on-board instalment & $\$ 1600$ & $\$ 1300$ \\
Second telehealth on-board instalment & $\$ 3200$ & $\$ 2600$ \\
Total on-board incentive & $\$ 4800$ & $\$ 3900$ \\
\hline
\end{tabular}

Telehealth service incentives are available for each telehealth consultation in addition to the telehealth MBS items. Payments are calculated and paid quarterly based on the number of telehealth consultations physicians have conducted in that quarter. A bulk billing incentive is also available for physicians who bulk bill their telehealth consultations. Both the telehealth service incentive and the bulk billing incentive are accrued and paid into the bank account listed for the relevant provider number at the end of each quarter. Physicians should remember that incentive payments are processed after the claim for a telehealth consultation has been lodged by the specialist or patient and any delay in lodging a claim will delay incentive payments.

\begin{tabular}{lcc}
\hline Incentive & $2012-13$ & $2013-14$ \\
Telehealth service incentive & $\$ 48$ & $\$ 39$ \\
Telehealth bulk billing incentive & $\$ 16$ & $\$ 13$ \\
\hline
\end{tabular}

More information on MBS items can be found on the MBS website, www.mbsonline.gov.au.

For more information please visit www.racptelehealth. com.au. 